\title{
NONLINEAR INTEGRAL EQUATIONS IN A MEASURE SPACE
}

\author{
P. C. DAS
}

\begin{abstract}
An abstract nonlinear integral equation is formulated in a measure space and existence, uniqueness of its solution is proved. It is shown that it includes as particular cases certain classes of integral equations and also differential equations containing measures.
\end{abstract}

This is an attempt towards defining a notion for the solutions of an equation which is an abstract formulation of a class of nonlinear integral equations and to study the existence of a solution of that equation in a sense to be defined.

In $\S 1$, we consider this abstract formulation and give an existence theorem for the solution. In $\S 2$, a link between these equations and equations from which this abstraction was made is established. It is to be noted that differential equations containing measures considered in [1], [2], [4], [6] and their abstraction in [5] from the point of view of existence of solutions are particular cases of the class of equations considered here. The whole idea in this abstraction is to free the differential and integral equations of implicit Cartesian co-ordinate system.

1. Let $(X, S, \mu)$ be a $\sigma$-finite measure space. Let $E^{*} \in S$ and let $\Lambda$ denote the space of all real valued signed measures defined on $E^{*} \cap S$. Let $\Lambda_{E}=\{\lambda \in \Lambda \mid \lambda(F)=\lambda(E \cap F)\}$ for each $\dot{E}=E^{*} \cap S . \Lambda_{E}$, and in particular $\Lambda$, is a Banach space with total variation norm [3]. $L(E, \mu)$ will denote the space of integrable w.r.t. $\mu$ functions mapping $E$ to $R$ and $L(\mu)$, the space of integrable with respect to $\mu$ functions mapping $E^{*}$ to $R$.

Let $G$ be a subclass of sets of $S$ contained in $E^{*}$ such that $\bigcup(A \mid A \in G)=$ $E^{*}$ and $G$ generates the $\sigma$-algebra $E^{*} \cap S$ on $E^{*}$. We assume further that restriction of every real signed measure on $E^{*} \cap S$ to $G$ has a unique $\sigma$ additive extension to the $\sigma$-algebra $E^{*} \cap S$.

We shall term such a class of sets " $g$-class" for the measurable space $\left(E^{*}, E^{*} \cap S\right)$. Now $\Lambda_{E}(G)=\{\lambda \mid \lambda$ is a real signed measure on $G$ and its extension belongs to $\Lambda_{E}$ \} is a Banach space with total variation norm where the total variation of an element is calculated for its extension in $\Lambda_{E}$.

Received by the editors March 24, 1972 and, in revised form, November 27, 1972 and April 10, 1973.

AMS (MOS) subject classifications (1970). Primary 34A10, 45G99, 45N05.

(C) American Mathematical Society 1974 
Now we consider the following equation

$$
\lambda(E)=v(E)+\int_{E-E_{0}} f\left(x, \lambda_{x}\right) d \mu,
$$

where $\nu \in \Lambda ; \lambda_{x}: \Lambda \times X \rightarrow R ; f: X \times R \rightarrow R ; E_{0} \in G$.

Definition. A real signed measure $\bar{\lambda}$ defined on $\bar{E} \in G$, is said to be a solution of (1) in $\bar{E}$ if $\vec{\lambda}$ satisfies the equation (1) for all $E \in G \supset E \subset \bar{E}$. For this purpose $\bar{\lambda}(E)=\bar{\lambda}(E \cap \bar{E})$ for $E \in E^{*} \cap S$.

Now we are in a position to establish some theorems concerning the existence of solutions of equation (1). Let $\Lambda_{E}^{a}(G) \subset \Lambda_{E}(G)$ denote the set of all finite signed measures in $\Lambda_{E}(G)$ for which $V_{E-E_{0}}(\lambda-v) \leqq a$, where " $V_{E-E_{0}}$ " denotes the variation over the measurable set $E-E_{0}$. By $\Lambda_{E}^{N a}(G)$ we shall denote the set of all real signed measures from $\Lambda_{E}^{a}(G)$ which coincide with $v$ on $E_{0} \cap G$. It is easily seen that $\Lambda_{E}^{N a}(G)$ is a closed subset of the Banach space $\Lambda_{E}(G)$.

THEOREM 1 (LOCAL EXISTENCE). Let $f$ be such that $f\left(x, \lambda_{x}\right)$ is measurable for each fixed $\lambda$, in the set $\Lambda_{E^{*}}^{N a}(G)$, and there exists an integrable function $U$ in $E^{*}$ satisfying $\left|f\left(x, \lambda_{x}\right)\right| \leqq U(x)$ (for all $\lambda \in \Lambda_{E *}^{N a}(G)$ ). Suppose also that there exists a constant $N$ such that

$$
\left|f\left(x, \bar{\lambda}_{x}\right)-f\left(x, \lambda_{x}\right)\right| \leqq N V_{E^{*}-E_{0}}(\bar{\lambda}-\lambda) \text { for } \bar{\lambda}, \lambda \in \Lambda_{E^{*}}^{N a}(G) .
$$

Finally, let there exist a set $\bar{E} \in G$ such that $\int_{E-E_{0}} U(x) d \mu \leqq a$ and $N \mu\left(\bar{E}-E_{0}\right)<1$. Then there exists a unique solution of the equation (1) on $\bar{E}$.

REMARK 1. The last condition is automatically satisfied if the measure is nonatomic or if the atoms are sufficiently small.

Proof. It is trivial to see that $\Lambda_{E}^{N a}(G)$ is a closed subset of the Banach space $\Lambda_{E}(G)$ with total variation norm. We denote by $T$ the following mapping from $\Lambda_{E}^{N a}(G) \rightarrow \Lambda_{E}(G)$

$$
(T \lambda)(E)=\nu(E)+\int_{E-E_{0}} f\left(x, \lambda_{x}\right) d \mu .
$$

The mapping is well defined on $\Lambda_{E}^{N a}(G)$. Now we verify that $T$ maps $\Delta_{E}^{N a}(G)$ into itself.

$(T \lambda)(E)=\nu(E)$ for $E \in G, E \subset E_{0}$ and consequently for $E \in S$ and $E \in E_{0}$. Further

$T$ is also a contraction.

$$
\begin{aligned}
V_{E-E_{0}}(T \lambda-v) & =V_{E-E_{0}}\left(\int_{E-E_{0}} f\left(x, \lambda_{x}\right) d \mu\right) \\
& \leqq \int_{E-E_{0}}\left|f\left(x, \lambda_{x}\right)\right| d \mu \leqq \int_{E-E_{0}} U(x) d \mu \leqq a .
\end{aligned}
$$

$$
\left(T \lambda^{1}-T \lambda^{2}\right)(E)=\int_{E-E_{0}}\left(f\left(x, \lambda_{x}^{1}\right)-f\left(x, \lambda_{x}^{2}\right)\right) d \mu .
$$


Hence

$$
\begin{aligned}
V_{E}\left(T \lambda^{1}-T \lambda^{2}\right) & \leqq \int_{E-E_{0}}\left|f\left(x, \lambda_{x}^{1}\right)-f\left(x, \lambda_{x}^{2}\right)\right| d \mu \leqq \int_{E-E_{0}} N V_{E-E_{0}}\left(\lambda^{1}-\lambda^{2}\right) d \mu \\
& =N \mu\left(\bar{E}-E_{0}\right) V_{E-E_{0}}\left(\lambda^{1}-\lambda^{2}\right),
\end{aligned}
$$

or $\left\|T \lambda^{1}-T \lambda^{2}\right\| \leqq k\left\|\lambda^{1}-\lambda^{2}\right\|$ where $k=N \mu\left(\bar{E}-E_{0}\right)<1$. So $T$ has a fixed point which is the required solution and the solution is unique.

2. In the following, we show that the above abstract equation has the following systems of equations as special cases.

$$
x(t)=h(t)+\int_{t_{0}}^{t} f\left(s, \int_{t_{0}}^{s} k(s, \xi, x(\xi)) d \xi\right) d g(s),
$$

where $h$ is a function of bounded variation in $\left[t_{0}, t_{1}\right], g$ is a right continuous monotonic increasing function $f: R^{2} \rightarrow R$, and $k: R^{3} \rightarrow R$.

$$
x(t)=h(t)+\int_{t_{0}}^{t} f(s, x(s)) d g(s),
$$

with $h, f, g$ as above.

$$
\lambda(E)=\lambda_{0}(E)+\int_{E-E_{0}} f\left(x_{1}, x_{2}, \lambda_{x_{1}, x_{2}}\right) d x_{1} d x_{2},
$$

where $E$ is a Borel measurable set in $R^{2}$ (Euclidean plane), and $\lambda_{x_{1}, x_{2}}$ : $\Lambda^{2} \times R^{2} \rightarrow R$, where $\Lambda^{2}$ denotes the class of real signed measures defined on Borel sets of $R^{2}$.

Lastly, the equation (1), also includes the equation considered in [5] as a particular case where $E^{*}=S_{\xi}$ in the notation of (5) and $\lambda_{x}=\lambda\left(S_{x}\right)$, a particular case of the mapping considered here. We verify our statement for the equation (1) only and others are similarly verified.

Consider $E^{*}=\left[t_{0}, t_{1}\right], E_{0}=\varnothing, \mu$ is the Lebesgue-Stieltjes measure generated by a right continuous monotonic function defined on $\left[t_{0}, t_{1}\right]$.

We have the equation

$$
x(t)=h(t)+\int_{t_{0}}^{t} f\left(s \int_{t_{0}}^{s} k(s, \xi, x(\xi)) d \xi\right) d g(s) .
$$

We see that the class of sets $F=\left\{\left[t_{0}, t\right]: t \in\left[t_{0}, t_{1}\right]\right\}$ is a "g-class". $S(F)$, the $\sigma$-ring generated by $F$, is precisely the class of Borel sets of $\left[t_{0}, t_{1}\right]$. It is also easily verified that a real signed measure defined on the Borel sets $\left[t_{0}, t_{1}\right]$ restricted to $F$ has a unique $\sigma$-additive extension to the Borel sets of $\left[t_{0}, t_{1}\right]$. We note that we can consider $\left[t_{0}, \infty\right)$ instead of $\left[t_{0}, t_{1}\right]$. 
Now let the equation (2) have a solution $x$ of bounded variation. Then,

$$
\begin{aligned}
x(t)-x\left(t_{0}\right)= & h(t)-x\left(t_{0}\right) \\
& +\int_{t_{0}}^{t} f\left(s, \int_{t_{0}}^{s} k\left(s, \xi, x(\xi)-x\left(t_{0}\right)+x\left(t_{0}\right)\right) d \xi\right) d g(s),
\end{aligned}
$$

where $k(s, \xi, y)=0$ for $\xi>s$. Every function of bounded variation $y(t)$ on $\left[t_{0}, t_{1}\right]$ defines uniquely a signed measure $\lambda$ on the Borel sets of $\left[t_{0}, t_{1}\right]$ with $\lambda\left(\left[t_{0}, t\right]\right)=y(t)-y\left(t_{0}\right)$. So we rewrite the equation (5) as

$$
\lambda\left(\left[t_{0}, t\right]\right)=v\left(\left[t_{0}, t\right]\right)+\int_{t_{0}}^{t} f\left(s, \lambda_{s}\right) d g(s)
$$

where $v\left(\left[t_{0}, t\right]\right)=h(t)-x\left(t_{0}\right)=h(t)-h\left(t_{0}\right)$ (taking into account the right continuity of $g$ ) and

$$
\lambda_{s}=\int_{t_{0}}^{s} \bar{k}\left(s, \xi, \lambda\left(\left[t_{0}, \xi\right]\right)+h\left(t_{0}\right)\right) d \xi .
$$

Hence writing $E=\left[t_{0}, t\right], E_{0}=\varnothing$, we have the following equation

$$
\begin{aligned}
& \lambda(E)=\nu(E)+\int_{E-E_{0}} f\left(s, \lambda_{s}\right) d g(s), \text { or } \\
& \lambda(E)=\nu(E)+\int_{E-E_{0}} f\left(s, \lambda_{s}\right) d \mu,
\end{aligned}
$$

putting $d \mu=d g(s)$. Thus every solution of (2) leads to a solution (6) which is an equation of the class (1).

Conversely if (6) has a solution, the steps can be retraced back and we get a solution of equation (2).

In fact we can formulate the following theorem for equation (2) for the existence of its solution. By b.v. we denote "bounded variation".

THEOREM 2. Let $h$ be b.v. function in $\left[t_{0}, t_{1}\right]$, and

$$
f\left(t, \int_{t_{0}}^{t} k(t, \xi, x(\xi)) d \xi\right)
$$

be measurable, for each function $x$ of b.v. belonging to the set $P=$ $\left\{y \mid V_{t_{0}}^{t_{1}}(x-h) \leqq a\right\}$. Suppose also that there exists an integrable function $U$ and $a$ constant $L$ such that

$$
\begin{gathered}
\left|f\left(t, \int_{t_{0}}^{t} k(t, \xi, x(\xi)) d \xi\right)\right| \leqq U(t) \\
|k(t, \xi, x(\xi))-k(t, \xi, y(\xi))| \leqq L|x(\xi)-y(\xi)|,
\end{gathered}
$$


for each $x, y \in P$. Let $|f(t, p)-f(t, q)| \leqq M|p-q|$ where

$$
p, q \in Q=\left\{y(t) \mid y(t)=\int_{t_{0}}^{t} k(t, \xi, x(\xi)) d \xi, \text { with } x \in P\right\} .
$$

Finally, let there exist a $t_{1}$ such that

$$
\int_{t_{0}}^{t_{1}} U(t) d g(t) \leqq a \text { and } L M\left(g\left(t_{1}\right)-g\left(t_{0}\right)\right)<1 .
$$

Then there exists a solution in $\left[t_{0}, t_{1}\right]$ for the equation (2).

Here, by a solution, we mean a function of bounded variation satisfying equation (2). We consider the equation (6), instead of equation (2). For equation (6) all the conditions of Theorem 1 are verified by maintaining $a$ and $U$ as such and putting $L M=N$. In fact,

$$
\begin{aligned}
\mid f\left(s, \bar{\lambda}_{s}\right) & -f\left(s, \lambda_{s}\right) \mid \\
& =\left|f\left(s, \int_{t_{0}}^{t_{1}} \bar{k}(s, \xi, \bar{x}(\xi)) d \xi\right)-f\left(s, \int_{t_{0}}^{t_{1}} \bar{k}(s, \xi, x(\xi))\right) d \xi\right| \\
& \leqq M\left|\int_{t_{0}}^{s} k(s, \xi, \bar{x}(\xi)) d \xi-\int_{t_{0}}^{s} k(s, \xi, x(\xi)) d \xi\right| \\
& \leqq M \int_{t_{0}}^{s}|k(s, \xi, \bar{x}(\xi))-k(s, \xi, x(\xi))| d \xi \leqq M L \int_{t_{0}}^{s}|\bar{x}(\xi)-x(\xi)| d \xi .
\end{aligned}
$$

Since $\bar{x}$ and $x \in \Lambda_{\left[t_{0}, t^{*}\right]}^{N a}(G)$, we have $\bar{x}\left(t_{0}\right)=x\left(t_{0}\right)=h\left(t_{0}\right)$ and hence,

$$
|\bar{x}(\xi)-x(\xi)| \leqq V_{t_{0}}^{t_{1}}(\bar{x}-x)=V_{t_{0}}^{t_{1}}(\bar{\lambda}-\lambda) \text {. }
$$

So $\left|f\left(s, \bar{\lambda}_{s}\right)-f\left(s, \lambda_{s}\right)\right| \leqq M L V_{E-E_{0}}(\bar{\lambda}-\lambda)$ in the notation of Theorem 1 . Hence, Theorem 2 follows from Theorem 1 .

Finally, the author thanks the referee for helpful comments.

\section{REFERENCES}

1. P. C. Das, Existence of solutions of differential equation approach, An. Sti. Univ. “Al. I. Cuza” Iasi Sect. I a Mat. 17 (1971), 53-59.

2. P. C. Das and R. R. Sharma, On optimal controls for measure delay differential equations, SIAM. J. Control 9 (1971), 43-61. MR 43 \#656.

3. N. Dunford and J. T. Schwartz, Linear operators. I. General theory, Pure and Appl. Math., vol. 7, Interscience, New York, 1958. MR 22 \#8302.

4. W. W. Schmaedeke, Optimal control theory for nonlinear vector differential equations containing measures, J. Soc. Indust. Appl. Math. Ser. A Control 3 (1965), 231-280. MR 32 \#7288.

5. R. R. Sharma, An abstract measure differential equation, Proc. Amer. Math. Soc. 32 (1972), 503-510. MR 45 \#691.

6. R. R. Sharma and P. C. Das, Existence and stability of measure differential equations, Czechoslovak Math. J. 22 (97) (1972), 145-158.

Hahn-Meitner-Institut für KeRnforschung, Berlin, Germany 